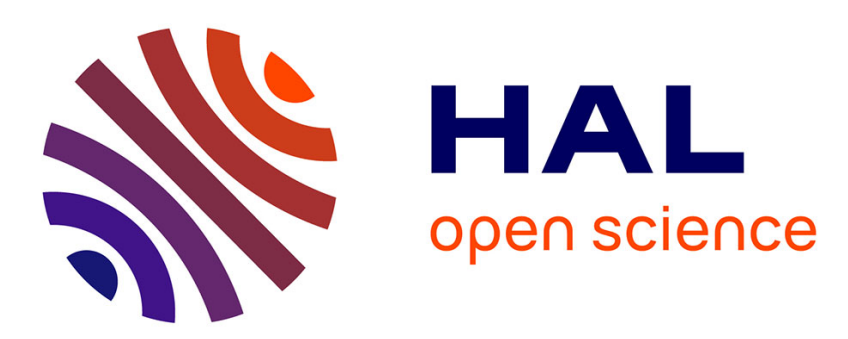

\title{
Scan Planning Strategy for a General Digitized Surface
}

Charyar Mehdi Souzani, François Thiebaut, Claire Lartigue

\section{To cite this version:}

Charyar Mehdi Souzani, François Thiebaut, Claire Lartigue. Scan Planning Strategy for a General Digitized Surface. Journal of Computing and Information Science in Engineering, 2006, 10.1115/1.2353853 . hal-01226542

\section{HAL Id: hal-01226542 \\ https://hal.science/hal-01226542}

Submitted on 13 Nov 2015

HAL is a multi-disciplinary open access archive for the deposit and dissemination of scientific research documents, whether they are published or not. The documents may come from teaching and research institutions in France or abroad, or from public or private research centers.
L'archive ouverte pluridisciplinaire HAL, est destinée au dépôt et à la diffusion de documents scientifiques de niveau recherche, publiés ou non, émanant des établissements d'enseignement et de recherche français ou étrangers, des laboratoires publics ou privés. 


\title{
Scan planning strategy for a general digitized surface
}

Charyar Mehdi-Souzani ${ }^{1}$, François Thiébaut ${ }^{1,2}$, Claire Lartigue ${ }^{1,2^{*}}$

${ }^{1}$ Lurpa, ENS de Cachan, 61 av du président Wilson, 94235 Cachan cedex, France

2 IUT de Cachan, 9 av Division Leclerc, 94234 Cachan cedex, France

Email : name@lurpa.ens-cachan.fr

\begin{abstract}
As new functional requirements of products lead to the definition of more complicated shapes, reverse engineering is playing a more important role. The process consists in defining a CAD model of the object surfaces from the measurement of the real object. Reverse Engineering takes advantages of new advances in non-contact measuring systems leading to a representation of the surfaces as large clouds of points. Nevertheless, scanning without path planning may affect completeness and accuracy of the measured data

This paper addresses the problem of intelligent scan planning within the context of reverse engineering. A measuring system allows us to acquire a cloud of points, which represents the first measurement of the free-form object. This incomplete and locally inaccurate cloud of points is used as a basis to generate an intelligent scan planning.

A pre-treatment of the point cloud is performed to determine the quality of the first scan and to find out the characteristic edges. The method relies on a voxel representation of the data. According to given thresholds of quality criteria (noise and completeness), unsatisfactory quality zones and digitizing gaps are identified. The new scan paths for an optimal digitizing are then calculated including optimal orientation search. An experimental application of the presented work is described through the digitising of a face mask.
\end{abstract}

Keyword: Reverse engineering, Quality of digitized data, Extraction of 3D edges, Sensor path planning

\section{Introduction}

Reverse Engineering (RE) of object surfaces is the process which constructs a CAD surface model from points derived from a measurement of an existing physical object. As new functional requirements of products lead to the definition of more complicated object shapes, reverse engineering is playing a more important role in the fields of moulds and dies for automotive and aeronautics industries [1]. Henceforth, there is a great interest in RE to obtain a numerical model of industrial parts for which the CAD model is unknown. RE consists of 3 main steps: part digitization, feature extraction, surface reconstruction or CAD modelling [2,3]. After the measuring step, the surface is known as a large cloud of 3D independent points which are usable for surface reconstruction. Most generally, data are pre-processed before exploitation in order to clean, filter, and reorganize the inhomogeneous and dense cloud of points [3].

Henceforth laser scanner sensors are largely used for part acquisition in RE as they are well-adapted for flexible, fragile and large size free-form surfaces. An essential issue is to define scan path planning in order to decrease manual operations and to have active control over scanning coverage. Scanning without path planning may affect completeness and accuracy of the data at the neighbourhood of edges, styling lines... essential in reverse engineering [4]. Indeed, the cloud of digitized points is the support for CAD modelling and numerous methods of surface reconstruction rely on finding surface boundaries such edges, styling lines or hole boundaries [5,6]. Some automatic methods have been developed to extract characteristic lines from discrete data. Due to the inaccuracy of 3D data and the lack of information at the neighbourhood of sharp edges, methods generally fail when applied to points issued from a real digitizing.

Most authors have addressed the problem of scan path planning for automated inspection. Lee and Park [7] automatically calculate scanning parameters (scanning direction, number of scans, scan path) from the CAD model of the part surface. They base their approach on a meshing of the parametric surfaces and calculate for each point of the meshing the local admissible direction (LAD) considering laser-scanning constraints such as the field of view, the depth of view (DOF), the view angle, ..... Resulting scanning directions also called globally accessible directions (GAD) are obtained by performing Boolean operations among LADs. The measuring system used relies on a 3-axis rotary table in order to set-up the part according to each scanning direction. Son et al [8] add to the previous work, the concept of critical points in order to decrease the number of scanning direction. Critical points are points that cannot be scanned together. Xi and Shu [4] formulate the problem as finding optimal settings of the scanning window (FOV) to maximize

\footnotetext{
* Corresponding author
} 
surface covering. They introduce slicing of the CAD model in order to divide the surface into sections, each of which can be covered by a scan pass. For a sliced section, the surface profile is obtained by projecting the surface onto the slicing plane. To find the optimal location of the FOV relative to the surface profile, they set the top of the FOV in alignment with the upper boundary of the surface profile. They define the coverage region by offsetting the upper boundary by the value of the depth of view. If the lower boundary of the surface profile belongs to the coverage region, then a whole surface section scanning is guaranteed. Bernard and Véron [9] have concentrated on the visibility theory applied to automated scan planning. The part visibility is calculated from a STL model of the surface. They introduce the concepts of global and local visibility with the objective of finding the minimum number of part set-ups so that the surface is completely scanned.

Prieto et al [10] focus on the definition of a scan planning strategy that also optimizes accuracy of the $3 \mathrm{D}$ points. Their objective is to produce a complete set of points with a predefined accuracy. Indeed they show that the digitizing noise is function of the incidence angle and the depth of view. In particular, noise is strongly reduced when the laser-beam is as normal as possible to the surface for a specific distance range. Authors compute a set of viewpoints for each point obtained from a $2 \mathrm{D}$ bitmap of the parametric surface. A viewpoint defines the optimal relative situation sensor/surface (spatial placement and orientation of the laser beam) so that the view angle $\alpha$ and the scanning distance $d$ remain within admissible ranges. A voxel representation of the points allows authors to define a collision-free scan path. Massios and Fisher [11] have also introduced a criterion of quality to automating the data acquisition process. The choice of the best-next view is performed according to a weighted sum of the visibility and the quality criteria. They take advantage of a voxel representation in particular to bring out unseen points.

Among the few works related to RE, the one by Zexiao et al [1] is particularly interesting. Indeed, they handle the whole problem of complete measurement in RE, highlighting the importance of accurate measurements of edges. The measuring system used is a Coordinate Measuring Machine (CMM) equipped with multi-probes: a structured light sensor and a trigger probe. The relative situations sensor/part surface are realised thanks to a rotary table. The light sensor is used for a first scan from which a CAD model is defined. The only constraint for the first scan is to lead to a complete object scanning. Edges are obtained from the CAD model by surface intersections. Regions requiring accurate measurement are re-measured using the trigger probe. Factors that may affect data accuracy are reviewed, leading to assess in particular sensor accuracy.

In this paper, a method for intelligent scan planning is proposed for smooth free-form surfaces within the context of RE. As the CAD model of the free-form surfaces is unknown, an arbitrary scan of the physical object is performed. The resulting cloud of points plays the role of the CAD model for automated accurate scanning with a specific attention to edges and styling lines. Our approach consists of two main stages: data pre-processing and intelligent scan-path planning which are detailed respectively in section 3 and 4 . Prior to the development of the approach, it is essential to clearly define the measuring system we use and its characteristics. Basically, data obtained using laser-sensors are dense, inhomogeneous and inaccurate which imposes the stage of data-pre-processing giving data a structure. As the first scan is carried out with an arbitrary relative setting sensor/surface, data quality can be evaluated in terms of quality indicators. Intelligent scan planning refers to defining a set of sensor settings so that the completeness is ensured and the given quality is reached, in particular for edges and surface contours.

\section{Characteristics of the digitizing system}

The 3D digitizing system used relies on a Coordinate Measuring Machine (CMM) equipped with a laser-plane sensor mounted on a motorized indexing head which enables the sensor to be oriented according to repeatable discrete orientations. As for common contact probes, orientations of the sensor are given by two rotations, the angles of which are A and B (figure 1). Due to the technology of the head, the number of rotations is limited: A and B can only be incremented every $7.5^{\circ}$. When the orientation is defined, the sensor can be moved according to the three translation axes of the CMM. Such a configuration increases the sensor's accessibility space and the use of a rotary table to locate the sensor relatively to the part surface becomes not necessary.

The sensor consists of a laser plane beam which is projected onto the part to be measured. The intersection of the part surface and the laser plane gives a curve which is observed in the 2D space of a CCD camera (Figure 1). Based on triangulation and pinhole modelling, the relationship between the 2D data acquired and the $3 \mathrm{D}$ coordinates of points belonging to the object surface is expressed [12]. 


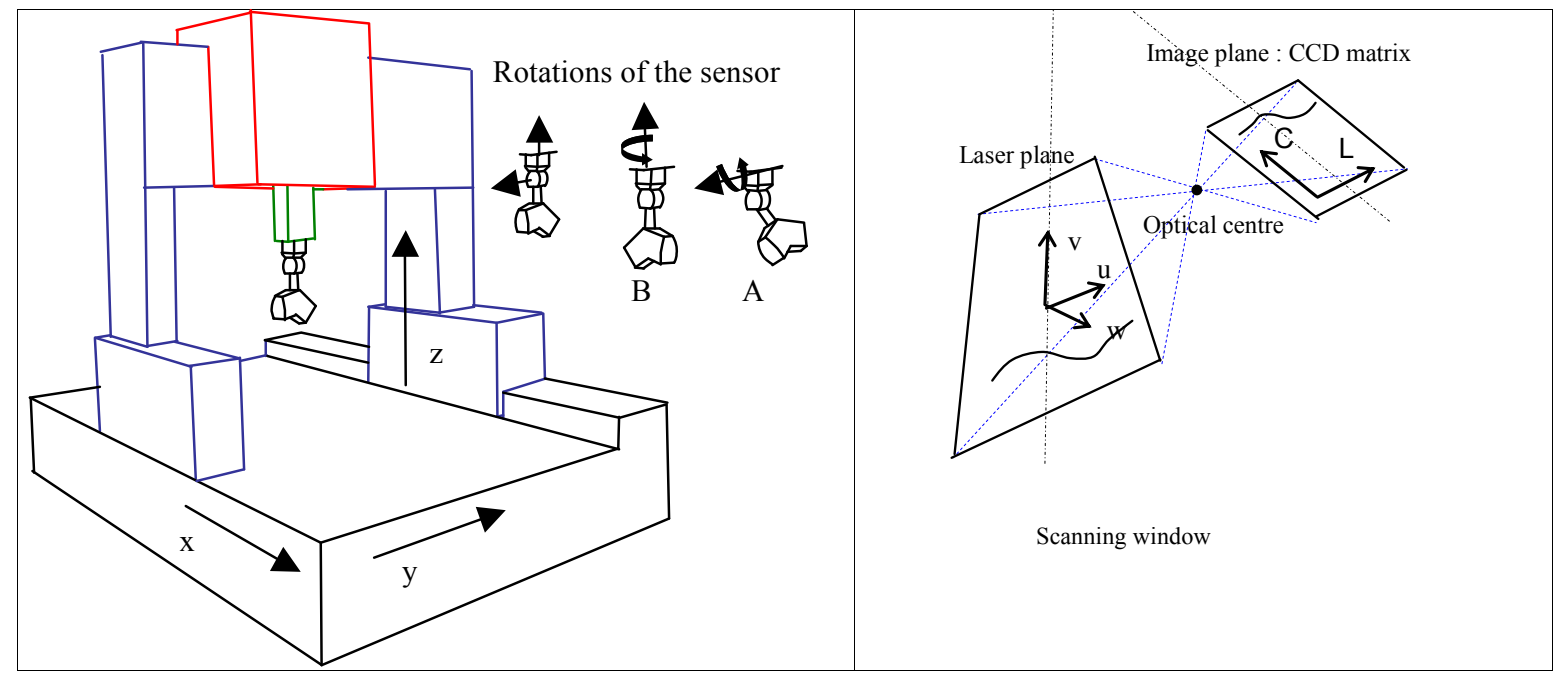

Figure 1: Digitizing system

To scan the whole surface, the sensor is moved in a certain direction. The sensor is characterized by a scanning window corresponding to the area of the scanning plane which is visible by the camera. This window, generally called field of view (FOV) is defined by the parameters $\mathrm{P}$ and L, where $\mathrm{P}$ is the effective height of the scanning plane and $\mathrm{L}$ is its maximal width. Only points of the intersection between the laser beam and the part surface belonging to the FOV are collected and define the digitized line. To accurately acquire the surface, the setting of the FOV as regards the part surface has to be optimal. This setting is dynamic as the surface geometry changes when the sensor is moved [4].

The optimal setting is most generally defined by the depth of view (DOF), distance between the measured point and the laser source, which should be within a specified range to ensure that the surface is fully covered [4,8]. It is henceforth admitted that accuracy of collected data is function of the incident angle, angle between the laser beam and the normal surface. Accuracy of the sensor decreases as the incident angle increases [8,13]. Smith and Zheng [13] propose to control the sensor orientation by the use of multi-laser displacement sensor but this procedure is specific.

Some authors focus on the evaluation of data quality with regards to the relative location sensor/surface in order to define optimal sensor settings [10,14-15].

Prieto et al [10] characterize the sensor quality in terms of digitizing noise, and concentrates on the evolution of the noise in function of the sensor positioning parameters $\alpha, \beta$, and $\mathrm{d}$ (figure 2). He shows in particular that the view angle, $\alpha$, is more influent than $\beta$, and must be less than $60^{\circ}$ to minimize the digitizing noise. This standpoint was adopted by Remy [16].

In previous works, we suggest evaluating the quality of digitized data through quality indicators and define four indicators: the density $\rho$, the completeness $\kappa$, the accuracy $\tau$, the noise $\delta[14,15]$. The first two indicators are intrinsic to the point cloud while the latter ones are inherent to the scanning process. The most relevant indicators to qualify digitized data for RE are the completeness and the noise [15]. The completeness ensures the validity of a part digitizing as regards topology and figures out the importance of the gaps existing in the point cloud. The noise $\delta$ is linked to data sampling errors and is generally evaluated considering the deviations between the points and a geometric model fitted to the points.

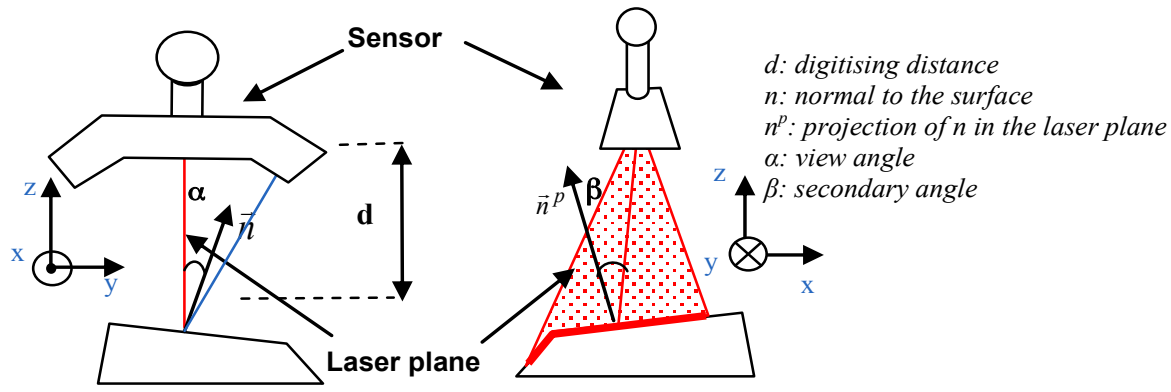


Recent works showed that the influence of the view angle, $\alpha$, and the digitizing distance $d$, on the noise $\delta$, when measuring a reference plane surface (figure 3) [17]. The evolution of $\alpha$ is performed for the recommended constructor distance value, $\mathrm{d}=150 \mathrm{~mm}$.

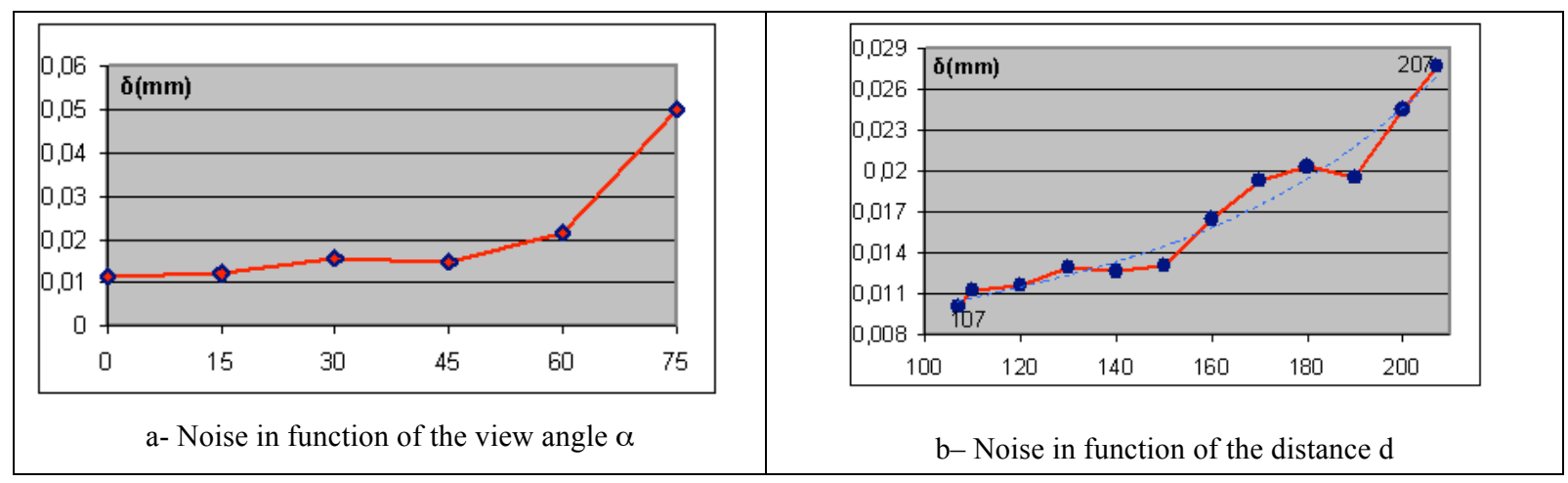

Figure 3: $\delta$-noise in function of the positioning parameters

In particular, figure 3a brings out two regions: one for which the noise is limited to $0.02 \mathrm{~mm}$ for $\alpha<60^{\circ}$ and the second one for which the noise increase drastically with $\alpha$. From this result, a range of admissible view angles can be defined according to the threshold value of $\delta$. Figure $3 \mathrm{~b}$ shows that the noise linearly increases with the distance $d$, for $\alpha=0^{\circ}$. It is interesting to notice that noise is not minimal for $d=150 \mathrm{~mm}$. Therefore, it would be appropriate to experiment the evolution of $\delta$ in function of $\alpha$ for $d=107 \mathrm{~mm}$.

Nevertheless, such results can be integrated in the approach of automated intelligent scan planning. Indeed, only when the sensor setting is correct as regards the digitizing distance $\mathrm{d}$ and the view angle, can the sensor scan with the given accuracy. To ensure the entire measurement of the part surface, several orientations are generally required. Whenever, the orientation is modified, a sensor calibration must be performed, resulting in loss of time and accuracy $[1,12]$. This leads to the following additional constraint: the number of orientations must be minimal.

The intelligent scan planning we develop leads to solve the following problem: finding the scan path defined from the minimum number of best sensor settings so that the completeness is ensured and the given precision is reached, in particular for characteristic lines.

Most of the methods that use the CAD model as support for finding the best settings rely on a sampling of the model through points and normals or through a STL model. In our approach, as the CAD model does not exit, we propose that a first scanning of the unknown surface plays the role of the sampled CAD model. To organize data and to be able to define local normal at the surface, we take advantage of a spatial representation based on voxels [18].

Therefore, the approach consists in a first scanning of the surface with a given sensor setting (figure 4). Data of this initial scan are pre-processed: voxel representation, quality evaluation and identification of characteristic lines. The quality of the point cloud is evaluated in terms of completeness and digitizing noise. According to a given threshold $\delta \max$, voxels which answer the criterion $\delta<\delta$ max are separated from those that do not answer it with a specific attention to the characteristic lines. 


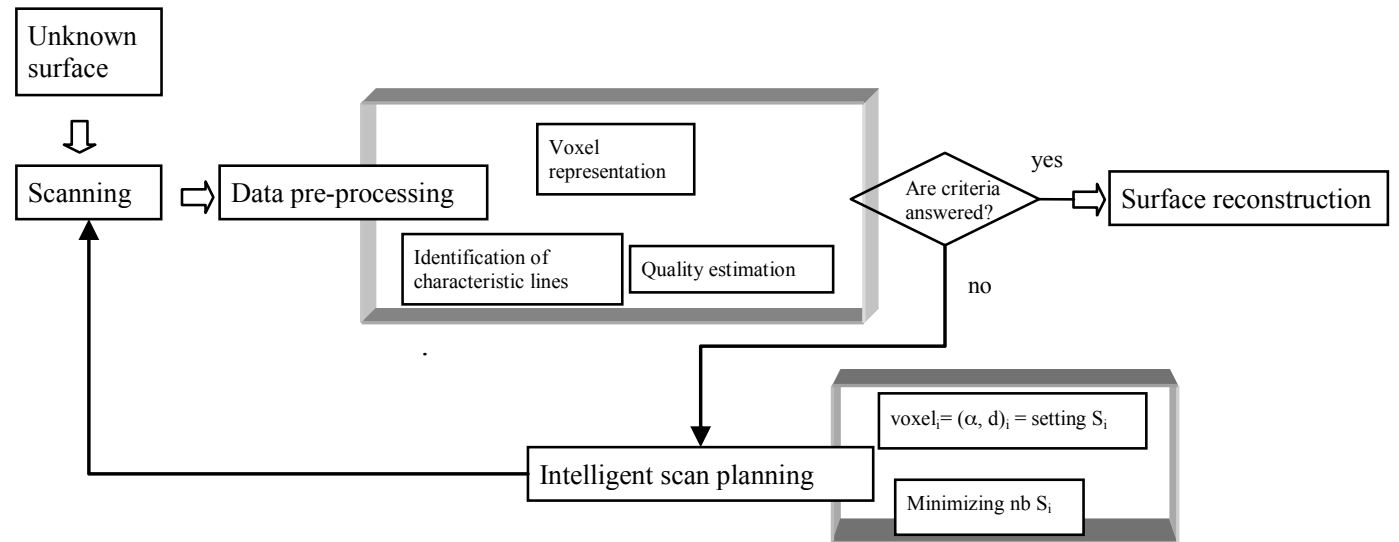

Figure 4: Overview of the approach

The completeness brings out the size of the digitizing gaps. Holes that exist in the object must be isolated from occlusion regions. For significant gaps and for regions which do not answer the expected quality an automatic calculation of the best settings is carried out. The objective is to ensure the completeness of the data as regards the real object surface and to guarantee high accurate measurements of characteristic zones while minimizing the number of orientation changes.

\section{Pre-processing of the first scan}

The first scan is performed manually by the operator, with the only objective to ensure the surface covering. The sensor is positioned according to sensor constructor requirements.

Therefore, the first setting of the sensor is chosen so that the normal the laser-plane remains parallel to the $\mathrm{x}$-axis or to the $\mathrm{y}$-axis. The simplest orientation corresponds to angles $\mathrm{A}=0^{\circ}$ and $\mathrm{B}=0^{\circ}$, that means that the laser plane is oriented by $y$-axis of the CMM. Therefore, the scanning direction is the $y$-axis, and the sensor is also set in different $x$ positions to ensure the whole surface coverage.

To test our approach, we use a white face mask, which is a 2,5D surface relatively smooth. This surface is voluntarily chosen simple in order to try out our approach for the identification of digitizing gaps which must be differentiated from actual holes of surface. In this direction, the surface topology presents some holes (face eyes) which must be clearly identified (figure 5).

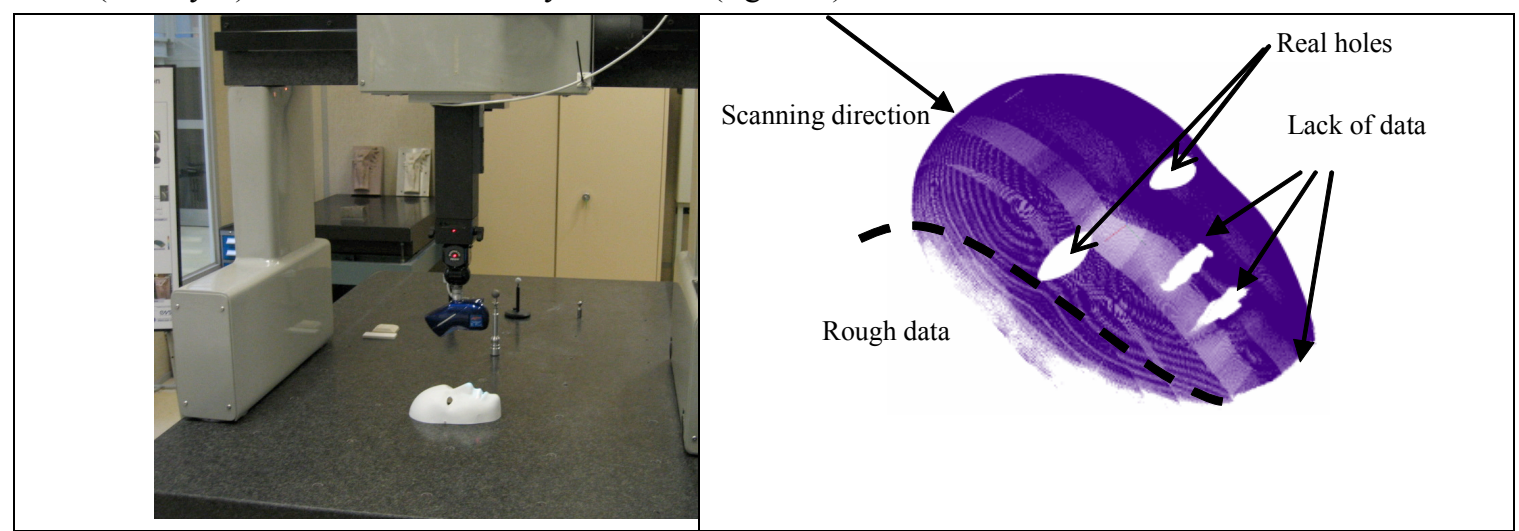

Figure 5: First scanning of the face mask

For the first scan presented in figure $5, \mathrm{~d}$ is chosen so that the top of the face nose is close to $100 \mathrm{~mm}$. As the depth of the mask is small enough, this involves the influence of the digitizing distance on the noise to be limited (see figure $3 \mathrm{~b}$ ). The laser stripe is about $35 \mathrm{~mm}$, so 8 positions on the $\mathrm{x}$-axis are necessary to cover the whole mask. The digitizing step is $0.1 \mathrm{~mm}$ which leads to about 900000 points. As expected, the cloud of points is dense, inhomogeneous and presents digitizing gaps which must be re-scanned. At this stage holes cannot be separated from occlusion regions and are treated in the same way. 
The first step of data pre-processing is data organisation through a voxel representation.

\subsection{Voxel representation}

The spatial 3D representation based on voxels gives the cloud of points a structure defining the notion of vicinity. A voxel can be defined as a 3D-pixel, and its usual representation is an elementary cube. In scan planning, voxels are generally classified in three types: non-empty voxels which contain a set of Mi measured points, empty voxel which dot not include points and unknown voxels the nature of which can not be determined [19].

We enrich this classification by giving attributes to non-empty voxels defined from the set of measured points belonging to the cube [18]. The most useful voxel attributes for intelligent scan planning are the voxel barycentre, $\mathrm{G}_{\mathrm{vox}}$ and the voxel normal, $\vec{n}_{v o x}$. If $\mathrm{N}_{\mathrm{voxj}}$ is the number of points belonging to the voxel $\mathrm{j}$, the barycentre is given by:

$$
\overrightarrow{O G}_{v x_{j}}=\frac{1}{N_{\text {voxj }}} \sum_{i=1}^{N_{\text {voxj }}} \overrightarrow{O M}_{i}
$$

The best least-square plane is fitted to the points belonging to the voxel $\mathrm{j}$ that defines the local normal $\vec{n}_{\text {voxj }}$ at the surface at the $\mathrm{G}_{\mathrm{voxj}}$. The orientation of each normal is outward material.

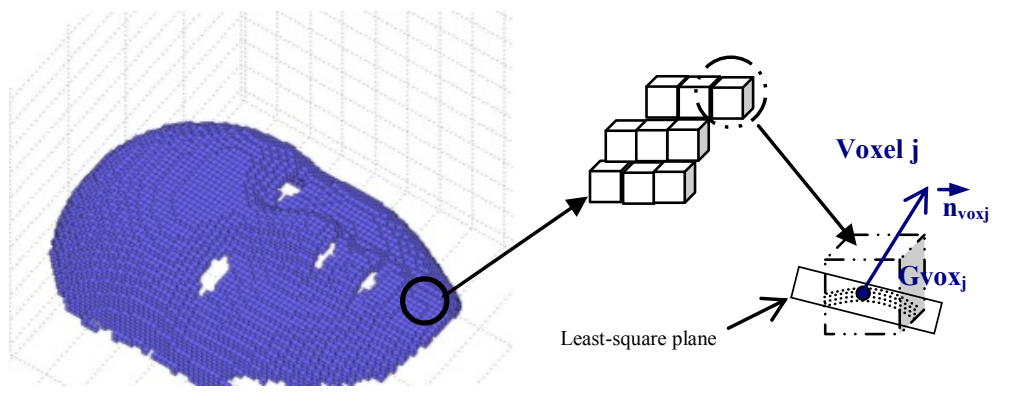

Figure 6: Voxel representation of the scanned mask

The voxel representation is applied to the data points of the mask, leading to 3524 voxels. The dimension of each cube is $4 \mathrm{~mm}$ and has been chosen so that each non-empty voxel contains a minimal number of points allowing the normal calculation. A structure thus exists giving the data a kind of pseudocontinuity as the notion of vicinity is available through voxel attributes and voxel neighbours. Furthermore, each voxel $\mathrm{j}$ can be substituted by the couple $\left(\mathrm{G}_{\mathrm{voxj}}, \vec{n}_{v o x_{j}}\right)$ of its attributes (Figure 6 ). This representation is used as support for the following stages.

\subsection{Analysis of data quality}

Due to the first arbitrary setting of the sensor relative to the surface, the view angle is not optimal for all the portions of the surface. As the digitizing distance is assumed to remain within the range $[100,150] \mathrm{mm}$, the evolution of the noise in function of $\alpha$ can be simply deduced from figure 3a. The laser-plane is oriented by $\vec{y}$ and the laser beam direction is $\vec{z}$, therefore for a voxel $\mathrm{j}$, the view angle $\alpha_{\mathrm{j}}$ is calculated according to:

$$
\cos \alpha_{j}=\frac{\left(\vec{n}_{\text {voxj }}-\left(\vec{n}_{\text {voxj }} \cdot \vec{x}\right) \cdot \vec{x}\right) \cdot \vec{z}}{\left\|\vec{n}_{\text {voxj }}-\left(\vec{n}_{\text {voxj }} \cdot \vec{x}\right) \cdot \vec{x}\right\|}
$$

A value of $\delta_{j}$ corresponds to each $\alpha_{j}$ and is affected to the voxel $j$. This defines the voxelmap of noise as illustrated in figure 7. 


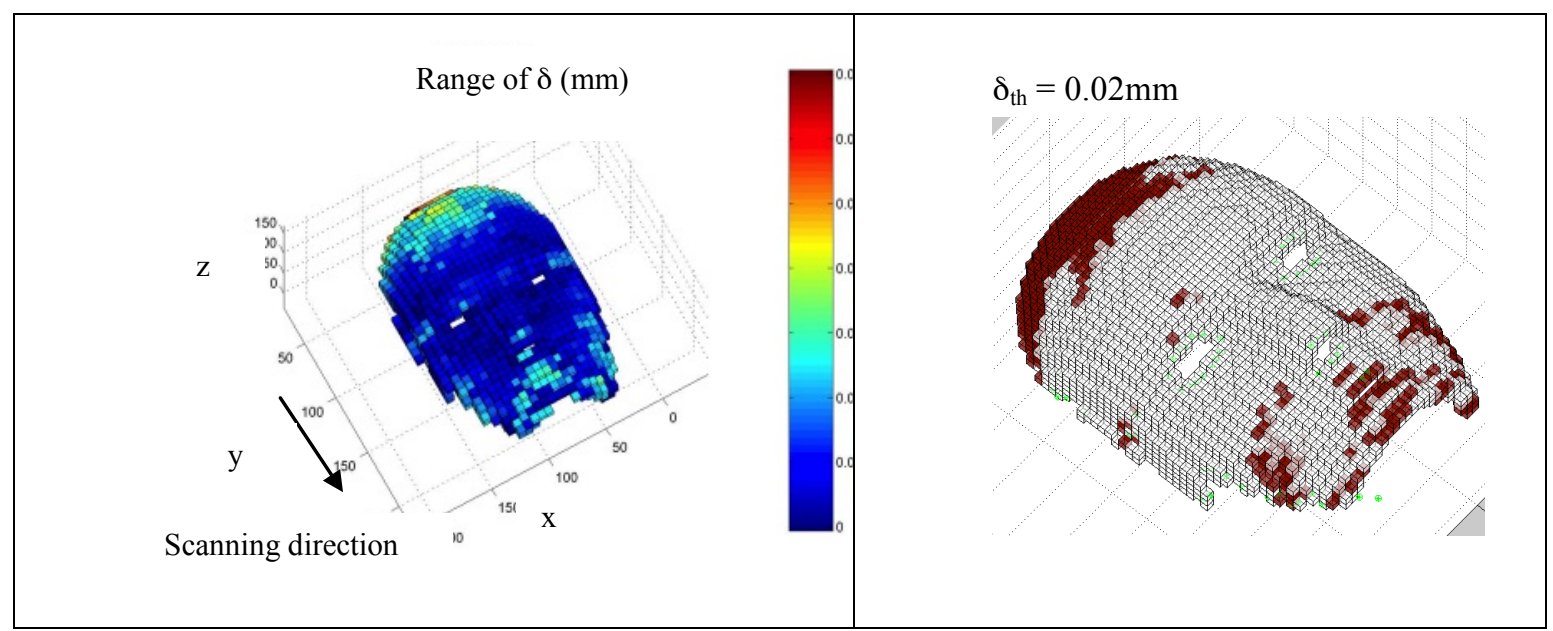

Figure 7: Voxel map of noise

As expected, the main part of the surface has a noise value within the range of 0.01 to $0.02 \mathrm{~mm}$. For surfaces quite vertical for which the laser-beam is almost parallel to the normal, the noise reaches values up to $0.07 \mathrm{~mm}$. In function of the given threshold $\delta_{\text {th }}$, the voxelmap is divided into 2 regions: one for which the quality criterion is answered, and another one which must be re-scanned to improve quality (decrease the value of $\delta$ ). The completeness allows the evaluation of the dimension of the gaps between neighbour points, and highlights areas for which holes are of a significant size. Nevertheless, we must not confuse a gap between two neighbour points with a hole. Completeness could be evaluated from a 2D Delaunay Triangulation DT of the digitised points [15].

Let $A_{F \mathrm{i}}$ be the calculated area of each triangular facet $F_{i}$ of the Delaunay mesh. To bring out digitizing gaps, we extract facets the area of which is greater than a threshold $A_{\max }$ :

$$
\left(\forall F_{i} \in D T, F_{i} \text { is a gap, if } A_{F i}>A_{\max }\right)
$$

As data are inhomogeneous, some facets can be of a stretched shape leading to small value of area although they present a large edge. At final, they can be missed although they are representative of gaps. Actually, this problem can be solved by calculating the Delaunay triangulation from the barycentres. The value of $A_{\max }$ is chosen relative to the mean value of the facet areas. For our study case the value of $A_{\max }$ is four times the mean value, $\mathrm{MV}=5.634 \mathrm{~mm}^{2}$. Data are thus analyzed, and digitizing gaps are identified (figure 8).
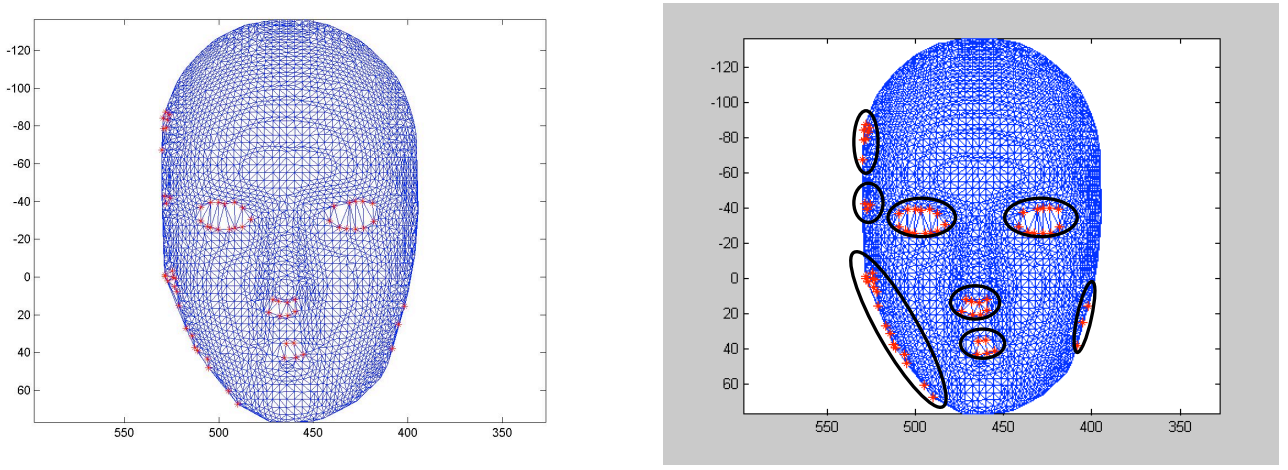

Figure 8: Completeness of the data for the face mask

Figure 8 presents the vertices of the identified large size facets (cross stars) defining regions with a lack of data. At this stage, the information does not allow us to conclude on the nature of these regions; they are identified as digitizing gaps for which re- scan is necessary. 


\subsection{Identification of the characteristic lines}

A characteristic line is defined as a set of characteristic points marking geometrical singularities $[6,18]$. Basically, this approach relies on a simple observation. When a plane cuts an object, a point of the contour can be identified by its singularities within the 2D profile: change in curvature, sharp angle, inflection point. As a result, the best cutting plane is that which is normal to the contour. Therefore, a smart sweeping of the cloud of points by cutting planes leads to the characteristic points of the 3D contours. Six types of characteristic points are defined which correspond to angular points, inflection points and extremities of the $2 \mathrm{D}$ profile. As extremity points define characteristic points, the boundary of a hole is a characteristic line.

The algorithm used to extract the points from discrete data is the directional coding. It relies on the polygonal approximation of the $2 \mathrm{D}$ points which are obtained by slicing the cloud of points by successive parallel planes, the direction of which is generally chosen as the scanning direction. If $\mathrm{p}$ is the digitizing step, the $2 \mathrm{D}$ points are obtained by projecting onto the slicing plane the points which distance to this plane is less than $\mathrm{p} / 2$. In order to only work on pseudo-continuous portions, the distance between two successive points is calculated. If this distance is greater than an admissible value, A, the profile is broken into 2 profiles which are separately analysed. This simply brings out hole edges, as profile extremities are characteristic points (Figure 9).

In order to filter the noise, 2D points are approximated by a set of segments according a chordal deviation criterion. Next, the coding algorithm analyzes the geometrical configuration of successive segments [6,18]. A code " 0 ", “+”, or "-" is affected to each segment depending on its length and its orientation as regards the previous one. For instance, for segments the length of which is greater than a given threshold, the corresponding code is " 0 ". For a segment which turns in the clockwise direction relatively to the previous one, the corresponding code is "-“; whether if turning in the counter clockwise direction, the code is "+" (Figure 9).

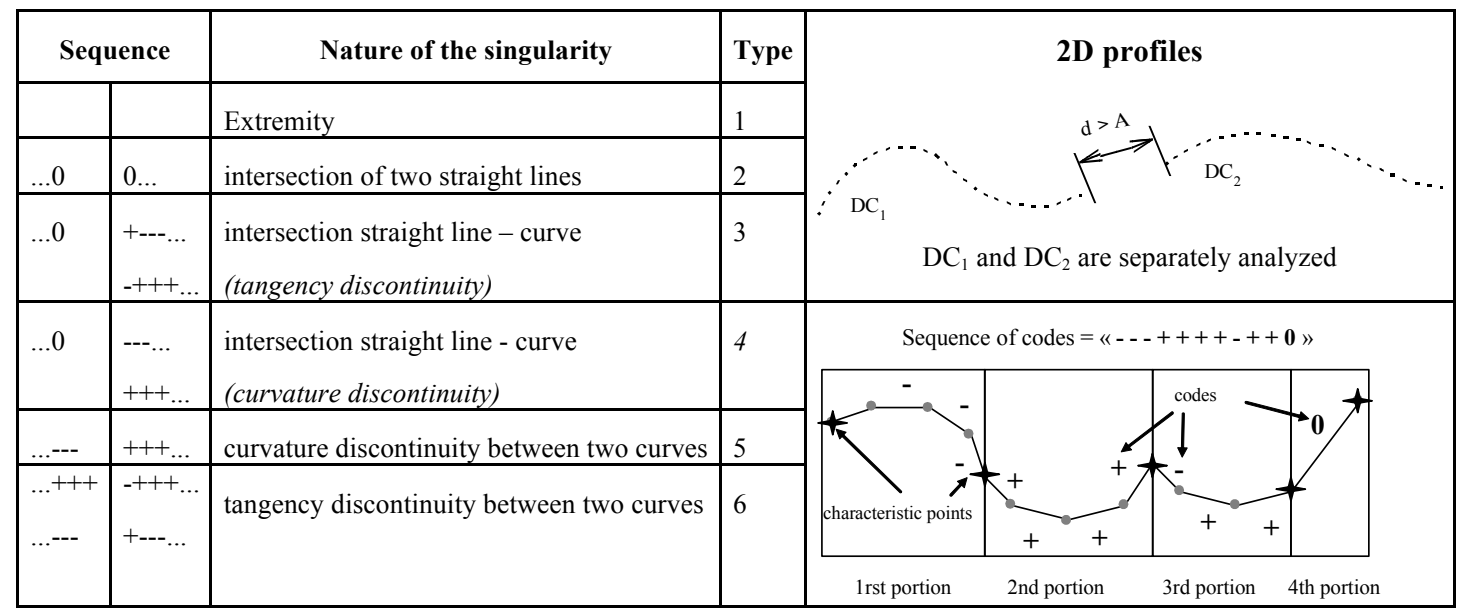

Figure 9: Identification of the characteristic points

The next stage is the identification of characteristic points which is performed through the analysis of the sequence of codes. Characteristic points represent geometrical singularities such as $\mathrm{C}^{1}$ or $\mathrm{C}^{2}$ discontinuities between portions and correspond to changes in the code value (figure 9). The example presented in the figure brings out five characteristic points.

As this method is time consuming when applied to the whole cloud of points, only barycentres are considered in a first approach. When the barycentre of a voxel is a characteristic point, the voxel is called a contour voxel. The construction of the characteristic lines is performed by the union of contour voxels according to proximity criterion and the concordance of the singularity nature. For instance, the edge of an actual hole is built by gathering contour voxels of type 1. For our example, the method is particularly efficient for hole edges (3D eye contours in our illustration) and part boundaries (figure 10). Results are coherent as regards completeness also highlighting the same digitizing gaps. 


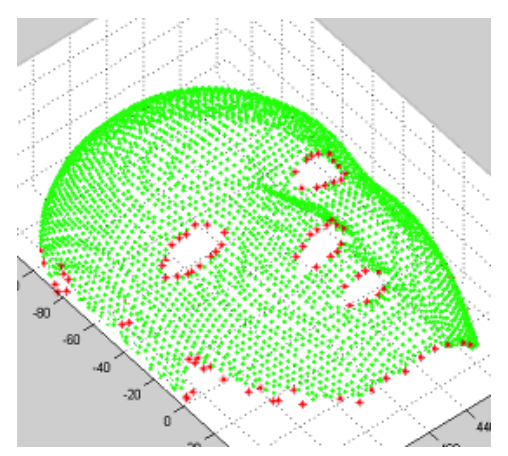

Figure 10: Characteristic points of type 1

Intelligent scan planning must lead to completeness of the data, separating actual holes present on the physical model from occlusion zones. Furthermore, the re-scan step allows better measurements of the characteristic lines by decreasing digitizing noise for such lines.

\section{$4 \quad$ Intelligent scan path planning}

The intelligent scan planning consists in determining the set of successive relative settings sensor/surface that lead to reach the quality of the digitizing and/or to complete the first scan. The displacement of the sensor is carried out thanks to the Numerical Controller (NC) of the CMM. For a given orientation of the sensor, the scan path is composed of a set of successive positions of the sensor. As the sensor is moved according to the scan path, points are acquired. Note that due to our sensor technology, the orientation remains fixed during the scanning. Prior to the definition of the path planning, we must expose the geometric model of the measuring system.

\subsection{Geometric Modelling of the measuring system}

Modelling requires the definition of coordinate systems attached to the different components of the measuring system [12].

Let us consider $\mathrm{R}_{\mathrm{c}}\left(O_{c}, \vec{x}, \vec{y}, \vec{z}\right)$ the frame linked to the CMM, and $\mathrm{R}_{\mathrm{s}}\left(O_{s}, \vec{u}, \vec{v}, \vec{w}\right)$ that linked to the sensor, for which $\mathrm{O}_{\mathrm{s}}$ is the origin of the frame, $\overrightarrow{\mathrm{w}}$ is normal to the laser plane, $\overrightarrow{\mathrm{v}}$ is the line symmetry of the scanning window and $\overrightarrow{\mathrm{u}}=\overrightarrow{\mathrm{v}} \otimes \overrightarrow{\mathrm{w}}$ (figure 11).

Let us call $\mathrm{m}$ the point defining the scanning path and $\mathrm{M}$ the digitized point. Coordinates $(\mathrm{X}, \mathrm{Y}, \mathrm{Z})$ of $\mathrm{M}$ are directly measured, and the position vector of $\mathrm{M}$ verifies:

$$
\overrightarrow{O_{c} M}=\overrightarrow{O_{c} m}+\overrightarrow{m O_{s}}+\overrightarrow{O_{s} M}
$$

where, the vector $\overrightarrow{O_{S} M}$ is the position of the point $\mathrm{M}$ expressed in $\mathrm{R}_{\mathrm{s}}$, defined by the coordinates $(\mathrm{u}, \mathrm{v}, \mathrm{w})$. The sensor setting is given by $\overrightarrow{O_{c} m}$. The coordinates $\left(\mathrm{x}_{\mathrm{s}}, \mathrm{y}_{\mathrm{s}}, \mathrm{z}_{\mathrm{s}}\right)$ of the vector $\overrightarrow{m O_{s}}$ expressed in $\mathrm{R}_{\mathrm{s}}$ are constant. Furthermore, the calibration step generally performed prior to the scan process, provides the list of vectors $(\overrightarrow{\mathrm{u}}, \overrightarrow{\mathrm{v}}, \overrightarrow{\mathrm{w}})$ corresponding to each possible orientation of the sensor (figure 11). Note that calibration allows point registration whatever the orientation used.

The automatic generation of the sensor path, that means the set of $\mathrm{m}$ points defining the path, is carried out from the voxel representation. We next detail the algorithm giving the intelligent scan path of a single voxel. 


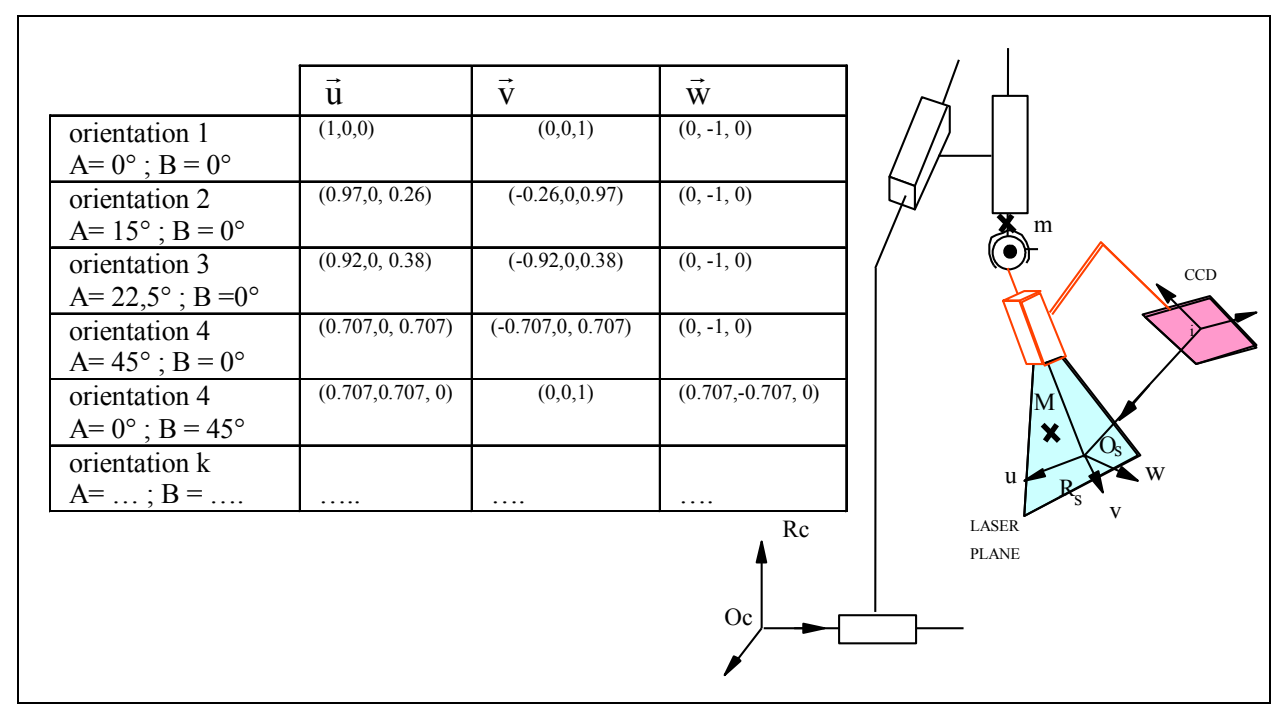

Figure 11: Representation of the geometric model

\subsection{Scan path for optimal digitizing of a single voxel}

The first scanning provides for each voxel, the couple of attributes $\left(\mathrm{G}_{\mathrm{vox}}, \overrightarrow{\mathrm{n}}_{\mathrm{vox}}\right)$. The objective here is to find $\overrightarrow{O_{c} m}$ so that the whole voxel can be digitized more accurately, that means finding admissible settings of the sensor relative to the given voxel under the followings constraints:

- The digitizing distance $d$ belongs to the admissible range: $d \in[100 \mathrm{~mm}, 150 \mathrm{~mm}]$

- The incident angle $\gamma$ belongs to the admissible range: $\gamma \in\left[0, \gamma_{\max }\right]$

- The digitized point belongs to the scanning window.

The incident angle $\gamma$ corresponds to a linear combination of $\alpha$ and $\beta$. In our application, we have considered $\beta$ of less influence than $\alpha$. We deduce that maintaining $\gamma$ within the range $\left[0, \alpha_{\max }\right]$ can be a correct assumption.

These three constraints which lead to find the set of $\left(\overrightarrow{m O_{s}} \text { and } P\right)_{\text {adm }}$ may be expressed relative to the $\mathrm{R}_{\mathrm{s}}$ coordinate as follows:

- As the distance $\mathrm{d}$ is directly linked to $\mathrm{v}$, imposing a range of distance is equivalent to impose an admissible range for $\mathrm{v}, \mathrm{v} \in\left[\mathrm{v}_{\min }, \mathrm{v}_{\max }\right]$

- The incidence angle is the angle between the normal to the voxel and $\vec{v}: \vec{n}_{v o x} \cdot \vec{v} \in\left[\cos \left(\gamma_{\max }\right), 1\right]$

- The digitized point belongs to the scanning window is assured while $u \in\left[\mathrm{u}_{\min }, \mathrm{u}_{\max }\right]$

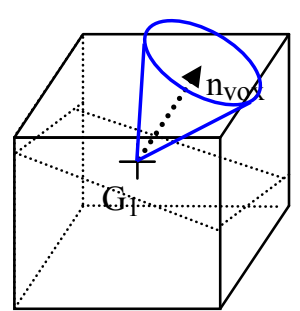

Admissible zone for $\mathrm{v}$

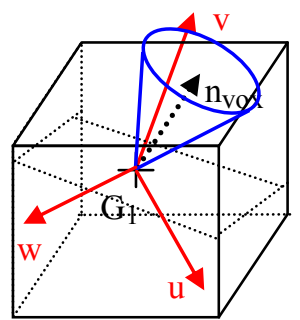

Selected orientation

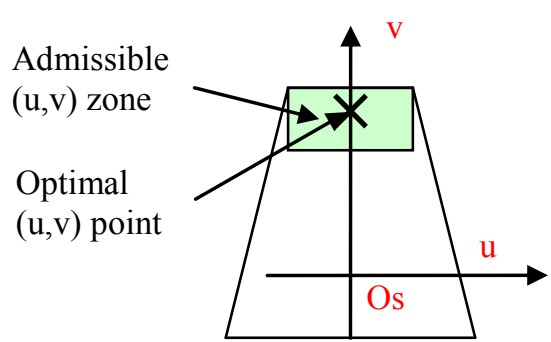

Figure 12: Representation of the voxel 
In order to respect these constraints, we have first to find out admissible orientations of the sensor among those possible. The set of admissible orientations permits to respect the constraint relative to the incidence angle: every rotation matrix that leads to respect $\vec{n}_{v o x} \cdot \vec{v} \in[\cos (\gamma \max ), 1]$ is admissible. When there is no additional constraint, the orientation that gives the minimal incidence angle is chosen. Once the orientation of the sensor is determined, its position is defined with respect to the optimal distance chosen between 100 and $150 \mathrm{~mm}$ which is imposes the value of v. Finally, the barycentre is set on the centre of the scanning window leading to $\mathrm{u}=0$.

The sensor is moved according to the $\overrightarrow{\mathrm{w}}$ direction to scan the whole voxel. Note that this method is efficient for voxels of small dimensions as regards the scanning window.

\subsection{Generation of the path for digitizing a characteristic line}

A characteristic line that needs to be re-scanned in order to complete a scanning or in order to increase the quality of the digitized points corresponds to a set of ordered voxels. Therefore, for each voxel, constraints exposed in the previous section must be respected.

Identification of characteristic points is most efficient when the $2 \mathrm{D}$ profile is normal to the characteristic line (see section 3.3). In order to ensure that the laser-plane is as much as possible normal to the characteristic line, we impose $\vec{w}$ to be collinear to the general direction of the line, defined by successive barycentre positions of the ordered voxels (figure 13).

For two successive voxels, the general direction is approximated by $\overrightarrow{G_{i} G_{i+1}}$, and the angle between the direction of the line and the plane normal is defined by:

$$
\cos \theta=\overrightarrow{\mathrm{w}} \cdot\left(\overrightarrow{\mathrm{G}_{\mathrm{i}} \mathrm{G}_{\mathrm{i}+1}}\right) /\left\|\mathrm{G}_{\mathrm{i}} \mathrm{G}_{\mathrm{i}+1}\right\|
$$

The new orientation constraint is imposed while imposing a minimum value to $\cos \theta$.

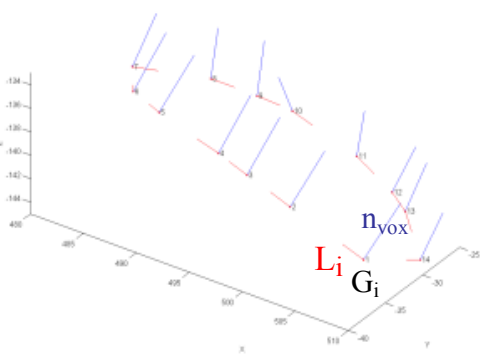

Attributes of edge contour voxels and characteristic line direction

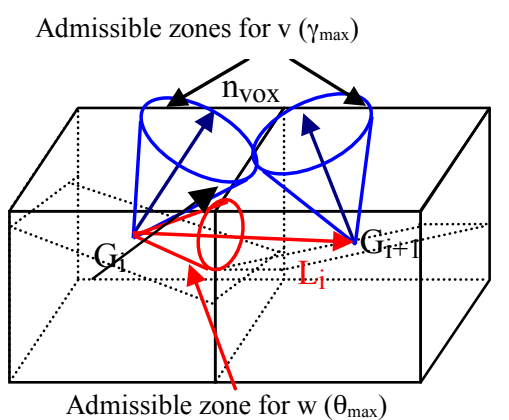

Admissible zone for $\mathrm{w}\left(\theta_{\max }\right)$

Figure 13: Representation of successive contour voxels

The steps of the path determination method are:

- Choice of the first point of the characteristic line $G_{1}$

- Calculation of the local direction of the line: $\overrightarrow{\mathrm{L}}_{\mathrm{i}}=\overrightarrow{\mathrm{G}_{\mathrm{i}+1} \mathrm{G}_{\mathrm{i}}} /\left\|\overrightarrow{\mathrm{G}_{\mathrm{i}+1} \mathrm{G}_{\mathrm{i}}}\right\|$

- Calculation of the admissible orientations of the sensor imposed to assure the scanning of all the voxels $\overrightarrow{\mathrm{n}}_{\mathrm{vox}} \cdot \overrightarrow{\mathrm{v}} \in[\cos (\gamma \max ), 1]$ and the constraints imposed by the line direction $\left|\overrightarrow{\mathrm{L}}_{\mathrm{i}} \cdot \overrightarrow{\mathrm{w}}\right| \in[\cos (\theta \max ), 1]$.

While all the constraints are respected, the next point of the line and the associated constraints is added. The resulting admissible orientation for the $\mathrm{n}$ considered voxels is then determined.

The $n$ successive positions of the sensor are then determined while imposing $\mathrm{u}$ and $\mathrm{v}$ so that the characteristic points are located at the optimal point of the laser plane.

This algorithm can be simplified for holes the dimension of which is small as regards the laser stripe. Indeed, imposing the laser-plane to remain as much as possible normal to the edge hole may lead to a high 
number of settings while a unique scan path may be convenient. Therefore, it is essential to compare the gap area to the area that can be swept by the laser stripe during a single path. This can be done by connecting the barycentres of the corresponding characteristic line and by calculating the area of the so built polygon. For small gaps, the 3D characteristic line becomes a limited plane. An illustration is given in the next section.

\subsection{Application}

For each region defined in figure 14, barycentres belonging to the edges of the digitizing gaps are identified. Let us consider one of these regions, region $i$. The edge is defined by the set $\{\mathrm{G}\}_{\mathrm{j}}^{\mathrm{i}}$, for which the best-fit least square plane is calculated giving its normal $\vec{N}^{i}$. The barycentre can also be calculated, $\mathrm{G}^{\mathrm{i}}$. Using the method presented in paragraph 4.2, the set of admissible orientations of the sensor is defined for each hole. Table 1 summarizes values of $\vec{N}^{i}$ for each region corresponding to digitizing gaps.

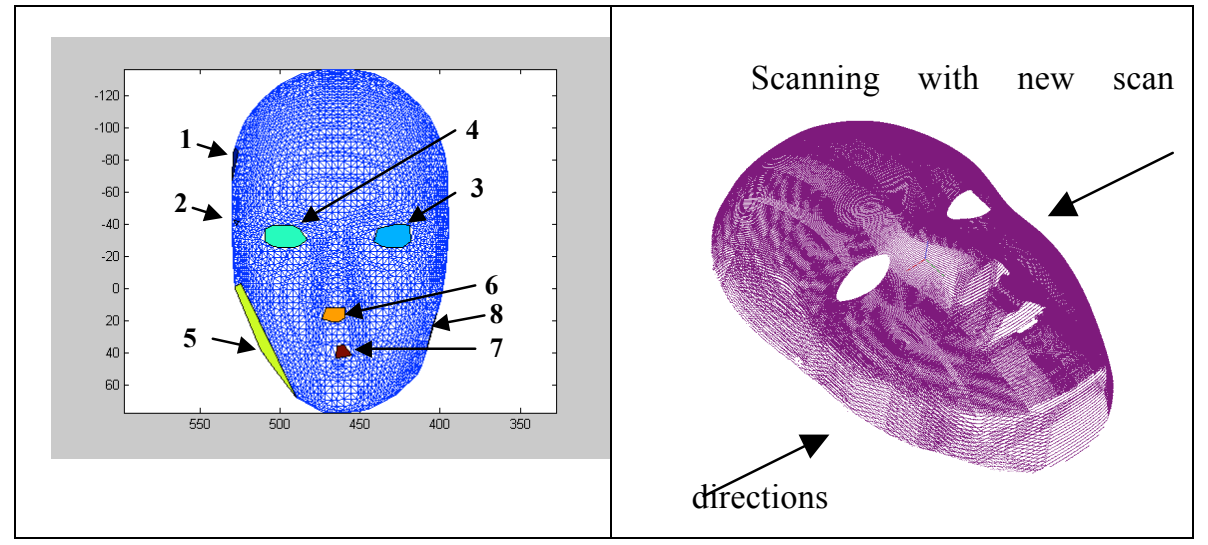

Figure 14: Result of the intelligent scanning

\begin{tabular}{|c|c|c|c|c|c|}
\hline $\mathrm{n}_{\mathrm{x}}$ & $\mathrm{n}_{\mathrm{y}}$ & $\mathrm{n}_{\mathrm{z}}$ & Zone \# & $\mathrm{A}$ & $\mathrm{B}$ \\
\hline 0,96 & -0.16 & 0.21 & 1 & $15^{\circ}$ & $-97.5^{\circ}$ \\
\hline 0.97 & 0.08 & 0.23 & 2 & $15^{\circ}$ & $97.5^{\circ}$ \\
\hline-0.26 & 0.27 & 0.92 & 3 & $105^{\circ}$ & $105^{\circ}$ \\
\hline 0.27 & 0.34 & 0.90 & 4 & $75^{\circ}$ & $112.5^{\circ}$ \\
\hline 0.90 & 0.39 & 0.18 & 5 & $15^{\circ}$ & $112.5^{\circ}$ \\
\hline 0.81 & 0.35 & 0.46 & 6 & $30^{\circ}$ & $112.5^{\circ}$ \\
\hline-0.95 & 0.30 & 0.13 & 7 & $0^{\circ}$ & $-105^{\circ}$ \\
\hline-0.16 & 0.78 & 0.61 & 8 & $105^{\circ}$ & $142.5^{\circ}$ \\
\hline
\end{tabular}

Table 1: Associated normal to each gap

Each calculated sensor orientation should result in a new digitizing process. Nevertheless, 8 new sensor settings are not compatible with the constraint of minimizing the number of orientations. For our study case we search the minimum number of orientations that permit to respect the constraints associated to the gaps. Two orientations of $\vec{v}$ appear necessary to re-scan all the digitizing gaps, orientations corresponding to region 6 and 7 (figure 14). After the intelligent scanning, the completeness is ensured: only actual holes of the mask (eyes and mouth) are present in the new digitized data (figure 14).

\section{Conclusion}

This paper presents a method for intelligent scan path planning within the context of reverse engineering of unknown surfaces. Our objective is to ensure the complete measuring of the object surfaces while respecting a quality criterion, in particular for characteristic lines of the surfaces.

The measuring system used is a CMM equipped with a laser-plane sensor mounted on a motorized indexed head, giving the sensor a set of fixed orientations. As the CAD model of the real part is unknown, our approach relies on a first scanning of the part surfaces. The acquired point cloud is modelled through a volumetric representation using voxel. Each voxel includes a surface portion to which attribute of normal and barycentre are calculated, and a portion of surface is substituted by its attributes. Contour voxels corresponding to characteristic lines are extracted from the initial point cloud. 
The intelligent scan planning leads to define optimal sensor settings ensuring the completeness of the data. A sensor setting is defined by its orientation within the CMM frame and its position vector. To answer quality criteria, the sensor setting is constrained by the view angle and the digitizing distance as regards each voxel, leading to admissible orientations among those accessible by the motorized head. Once the orientation of the sensor is determined, its position is defined, so that the barycentre of the digitized voxel is positioned on the symmetry axis of the scanning window. A characteristic line is a succession of contour voxels to which corresponds a succession of admissible settings, and the scan path is defined by assembling the successive settings.

The algorithm is simplified for digitizing gaps presenting a width small as regards the laser stripe. Our approach, illustrated through the example of a face mask leads to ensure completeness of the data at the neighbourhood of edges. Further works are in progress, in particular to assess the efficiency of the method for more complicated part geometries.

\section{References}

[1] Zexiao X., Jianguo W., Qiumei Z., 2005, “Complete measurement in reverse engineering using a multiprobe system", International Journal of Machine Tools and Manufacture, 45(12-13), pp. 1474-1486

[2] Chang D.Y., Chang Y.M., 2002, "A freeform surface modelling system based on laser scan data for reverse engineering”, International journal of Advances Manufacturing technology, 20, pp. 9-19

[3] Varady T., R. Martin R., Cox T., 1997, "Reverse engineering of geometric models - an introduction", Computer Aided Design, 29(4), pp 255-268

[4] Xi F., Shu C., 1999, “CAD-based path planning for 3-D line laser scanning”, Computer-Aided Design, 31 , pp. 473-479

[5] S. Azernikov, A. Fischer, 2003, "Surface Reconstruction of free form objects based on multiresolution volumetric method for distributed CAD”, CIRP Design Seminar 2003, Grenoble(France), 12-14 may 2003 (CD-Rom paper)

[6] G. Osty, C. Lartigue, 1998, "Partition of 3D digitized data for CAD modelling", International Journal of CAD/CAM and computers graphics, 13(4-5-6), pp. 263-272

[7] Lee K., Park H.P., 2000, "Automated inspection planning of free form shape parts by laser scanning", Robotics and Computer Integrated Manufacturing, 16, pp. 201-210

[8] Son S., Park H., Lee K.H., 2002, "Automated laser scanning system for reverse engineering and inspection", International Journal of Machine Tools and Manufacture, 42, pp. 889-897

[9] A. Bernard, M. Véron, 2000, "Visibility theory applied to automatic control of 3D complex parts using plane laser sensors", Annals of the CIRP, 49(1), pp. 113-118

[10] F. Prieto, T. Redarce, P. Boulanger, R. Lepage, 2000, “Accuracy Improvement of contactless sensor for dimensional inspection of industrial parts", International Journal of CAD/CAM - Rapid Production, 15, pp. 345-366

[11] Massios, N., Fisher R., 1998, “A best next view selection algorithm incorporating a quality criterion”, Proceedings of British Machine Vision conference, Southampton(England), pp. 780-789

[12] Lartigue C., P. Bourdet, L. Mathieu, C. Mehdi-Souzani, 2004, "Algorithms for the calibration of laser plane sensors on CMMs", Series on advances in Mathematics for applied sciences, 66, pp. 82-97

[13] Smith K.B., Zheng, Y.F., 1994, "Multi-laser displacement sensor used in accurate digitizing technique”, Journal of Engineering for Industry, 116, pp.482-483

[14] Contri A., 2002, "Qualité géométrique de la mesure de surfaces complexes par moyens optiques", PhD thesis, ENS de Cachan (France), November 2002

[15] Lartigue C., Contri A., Bourdet P., 2002, "Digitized point quality in relation with point exploitation", Measurement, 32, pp. 193-203

[16] Remy S., 2004, “Contribution à l'automatisation du processus d'acquisition de formes complexes à l'aide d'un capteur laser plan en vue de leur contrôle géométrique”, PhD Thesis UHP Nancy(France), Juin 2004

[17] Mehdi-Souzani C., Lartigue C., Thiébaut F., Bourdet P., 2005, "Evaluation de la performance des systèmes optiques de numérisation 3D - Application à la numérisation automatique intelligente", 3D Modelling, Paris(France), 27-28 septembre 2005 
[18] Osty G., 2001, "Extraction de particularités sur données discrètes issues de numérisation 3D: Partitionnement de grands nuages de points”, PhD Thesis, ENS de Cachan (France), January 2001

[19] Papadopoulos-Orfanos D., Schmidtt F., 1996, "Numérisation automatique avec un capteur 3D à profondeur de vue limitée", 5e Assises Européennes du Prototypage Rapide, 2-3 octobre 1996, Paris 